\title{
L'Association des facultés de médecine du Canada (AFMC) présente un nouveau prix international : le prix international AFMC-Charles Boelen pour la responsabilité sociale
}

\begin{abstract}
L e prix international AFMC-Charles Boelen pour $\mathcal{L}$ la responsabilité sociale soulignera des réalisations remarquables dans la mise en œuvre des principes de la responsabilité sociale dans le domaine de la santé. De par sa nature internationale, il visera à honorer des individus ou organisations de par le monde dont les réalisations sont des exemples d'application des principes de responsabilité sociale tels que définis par le Global Consensus for Social Accountability of Medical Schools (www.healthsocialaccountability.org) et autres références reconnues internationalement.
\end{abstract}

\section{Critères pour l'attribution du prix}

Le prix reconnaîtra une réalisation remarquable dans la mise en œuvre des principes de responsabilité sociale dans le milieu universitaire de la santé en démontrant :

- Une action centrée sur les besoins prioritaires de santé des citoyens et de la société dans l'accomplissement des missions d'éducation, de recherche ou de prestation de services, en accord avec les valeurs de qualité, équité, pertinence et efficience.

- Une démarche prenant en compte les besoins globaux de la personne ainsi que les déterminants sociaux de santé pour la population.

\section{Pentagone du partenariat}

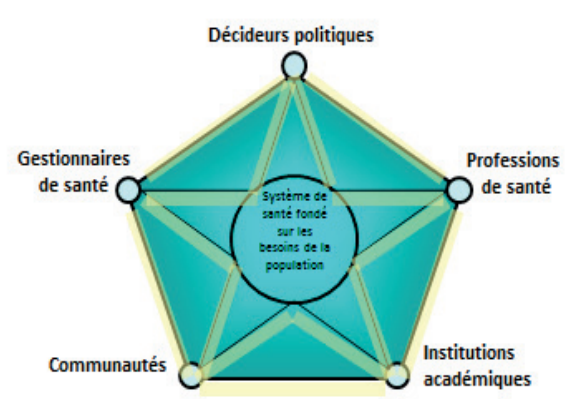

Boelen C. Towards unity for health, WHO, 2000.

- Un partenariat confirmé avec les principaux acteurs de santé pour une synergie dans le développement sanitaire.

- L'utilisation d'indicateurs et de mécanismes d'évaluation permettant de mesurer l'impact de l'établissement universitaire sur la performance du système de santé et le niveau de santé de la population.

- La contribution à l'élaboration de politiques nationales ou régionales tendant à soutenir l'établissement universitaire engagé dans des actions à grand impact sociétal.

Alors que le prix honorera un travail effectué dans l'un des trois domaines de l'éducation, de la 
recherche ou de la prestation de services, une préférence sera accordée à une œuvre impliquant ces trois domaines de façon intégrée. Le degré de reproductibilité dans d'autres établissements universitaires au niveau national, régional ou mondial sera également pris en compte.

\section{Admissibilité au prix}

Le prix pourra être attribué à une personne, une équipe, un département, un établissement ou une organisation dans le milieu universitaire de la santé. L'appel de candidatures se fera en même temps que pour les autres prix de l'AFMC, mais sera fait de manière très large par le truchement des moyens de communications appropriés pour permettre que l'on reconnaisse un travail exceptionnel accompli partout dans le monde. Dans le cadre du processus annuel de sélection, on étudiera des candidatures provenant de différents régions et pays du monde, afin que soit reflétée la diversité mondiale d'un point de vue géographique, culturel et linguistique.

\section{Processus de nomination et de sélection}

Les candidatures peuvent être soumises par une personne ou une organisation.

Les nominations doivent être soumises avant la date limite du 31 octobre pour le prix de l'année en cours. Le lauréat se verra remettre un globe de cristal, un certificat de 1000 \$ et une allocation pouvant aller jusqu'à 2000 \$ pour assister à la Conférence canadienne sur l'éducation médicale (CCEM) au cours de laquelle le prix sera habituellement remis. Les frais d'inscription à la conférence seront également couverts.

La documentation suivante devra être fournie pour chaque candidature : nom et affiliation, un CV s'il s'agit d'une personne ou la description des missions s'il s'agit d'une équipe, d'un département, d'un établissement ou d'une organisation, la description des travaux correspondants aux critères décrits plus haut, ainsi qu'une lettre de recommandation ne dépassant pas 1000 mots.

Une collaboration avec d'autres établissements universitaires, organisations ou associations de par le monde engagés à promouvoir les principes de responsabilité sociale au niveau international est fortement souhaitée durant tout le processus d'attribution du prix.

\section{À propos de Charles Boelen}

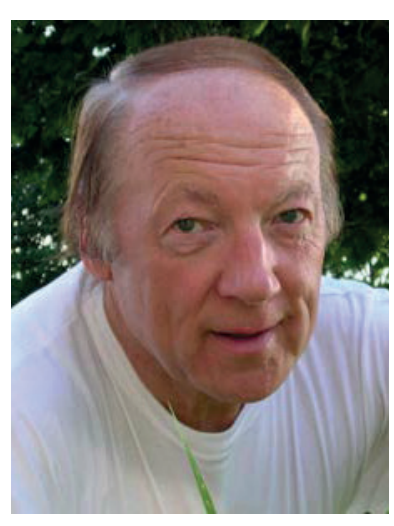

Charles Boelen est un médecin d'origine belge, spécialisé en santé publique, en gestion des systèmes de soin de santé et en éducation médicale. Il a une vaste expérience en santé internationale, dont 30 années au sein de l'équipe de l'OMS. Son travail porte sur les partenariats en santé, la responsabilité sociale des institutions d'enseignement et l'évolution des différentes professions en santé.

\section{À propos de l'Association des facultés de medecine du Canada (AFMC)}

L'Association des facultés de médecine du Canada (AFMC) représente les 17 facultés de médecine du Canada et est la voix nationale de la médecine universitaire. Notre organisme a été fondé en 1943 et appuie individuellement et collectivement les facultés de médecine canadiennes par le biais de la promotion de l'éducation médicale, de la recherche et des soins cliniques.

«La responsabilité sociale est au cœur de notre organisation et nous avons hâte de décerner ce prix pour la première fois lors de la Conférence canadienne sur l'éducation médicale (CCEM) l'an prochain à Vancouver ».

Docteure Geneviève Moineau, Présidente-Directrice Générale, AFMC

AFMC 265, avenue Carling, bureau 800, Ottawa (ON) K1S 2E1,

Canada 